\title{
STANDPUNKT
}

\section{Rote Karte gegen Zwangsprostitution - eine fragwürdige Kampagne des Deutschen Frauenrats}

\author{
Monika Frommel
}

S icher gibt es schreckliche Fälle von »Zwangsprostitution«. Aber alle ExpertInnen wissen auch, dass nicht der Zwang, schon gar nicht der physische Zwang zur Prostitution das Problem ist, sondern die ungeregelten Arbeitsbedingungen in der Prostitution, indirekter ökonomischer Zwang also: die Strukturen dieses Marktes, nicht nur die Brutalität von Zuhälter und Bordellbetreiber. Dass dabei eine irreguläre Migration die ohnehin reichlich vorhandenen Probleme verschärft, ist ebenfalls klar. Aber auch das ist kein spezifisches Problem von Prostituierten, sondern eines, das alle irregulären MigrantInnen teilen und das mit den erweiterten Strafbestimmungen gegen Menschenhandel vermutlich nicht gelöst werden kann. Liest man die dramatisierenden Texte, die mit der Kampagne des Frauenrats kursieren, dann wird deutlich, dass es im wesentlichen politische Korrektheit ist, welche es den kirchlichen und anderen Frauennetzwerken verbietet pauschal gegen den schwarzen, grauen und selbst dort, wo viel verdient wird, ungeregelten Markt sexueller Dienste zu wettern. Also suchen und finden sie Opfer. Aber mit dem Label »Zwangsprostituierte« kann man den Angesprochenen nur selten helfen und erfüllt wohl eher eine Mittelschicht spezifische Annahme, dass eine Frau nur aus Not und insoweit gezwungen so etwas macht.

2002 war das Ziel des ProstG die Lage der dort Tätigen zu verbessern, insbesondere sollte der Zugang zu den Sozialversicherungen ermöglicht bzw. erleichtert werden. Aber bis heute sind die Voraussetzungen für eine angemessene Kontrolle nicht geschaffen. Die entsprechenden gewerberechtlichen Genehmigungen werden nicht oder nur so erteilt, dass eben nicht sicher gestellt ist, dass Einnahmen versteuert, Abgaben bezahlt, Arbeitsverträge geschlossen und der arbeits- und mietrechtliche Schutz aktiviert wird. Man tut so, als seien alle Prostituierten frei beruflich tätig. Natürlich gibt es auch dieses, aber der Markt ist geprägt von organisierten Anbietern. Bis auf wenige Kommunen (Dortmund als positives Beispiel) blieb es also bei einer halbherzigen Legalisierung. Dem Abbau strafrechtlicher Interventionen stehen keine alternativen Kontrollen gegenüber. Dass die Polizei und die Strafverfolgungsorgane mit der EU-Erweiterung kaum noch Eingriffsbefugnisse haben, liegt auf der Hand. Aber die früher übliche polizeiliche und strafrechtliche Kontrolle der schlichten Förderung der Prostitution war nun einmal diskriminierend und konnte außerdem Zwang, Gewalt und Ausbeutung in der Prostitution nicht minimieren. Ausbeutung lässt sich nur feststellen, wenn Verträge existieren, Bücher geführt werden und die Polizei Zwangsbefugnisse hat, die dazu führen können, die ökonomischen Zusammenhänge zu durchleuchten. Dies ist bei einer Razzia nicht möglich und dies ist auch nicht die Aufgabe der Polizei.

Setzt man aber die Polizei unter politischen Druck, was ja der Sinn der Kampagnen gegen »Zwangsprostitution « ist, und besteht auf den klassischen Forderungen an die Strafverfolgungsorgane, »Zwang und Ausbeutung « zu verfolgen, dann handelt man sich ein unlösbares Dilemma ein: Ausbeutung lässt sich mit polizeilichen Mitteln nicht feststellen, wenn Finanzämter untätig geblieben sind. Zwang in und Zwang zur Prostitution ist strafrechtlich verfolgbar, aber nur dann, wenn Prostituierte Anzeigen er- statten, was voraussetzt, dass die Rechtstellung von Prostituierten der anderer ArbeitnehmerInnen / Selbständigen faktisch angenähert wird. Eine Kampagne, welche nach mehr Polizei und mehr Strafrecht ruft, die Lage der illegalen und sehr jungen Prostituierten. Sie hat nämlich zur Konsequenz, dass Prostituierte unter 18 Jahre (wegen $\S \S 180$ Abs. 3 und 180 a Abs. 3 Nr. 1 StGB) und nicht EU-Bürgerinnen auf den Straßenstrich abgedrängt werden, was ihre prekäre Situation eher verschärft als mindert. Es wäre daher sinnvoll, diese Strafvorschriften - $\S \S 180$ Abs. 3 und 180 a Abs. 3 Nr. 1 StGB - zu modifizieren, um jungen Prostituierten mit akzeptierender Sozialarbeit Unterstützung

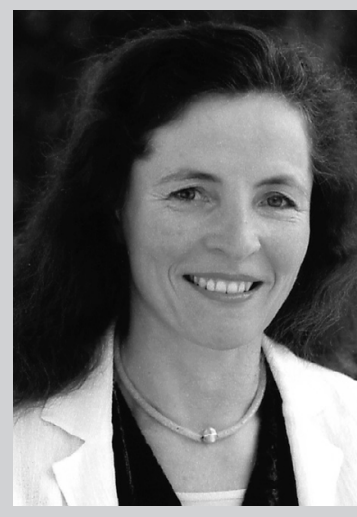

Prof. Dr. Monika Frommel ist Schriftleiterin dieser Zeitschrift gewähren zu können (Stichwort: Drogenstrich). Auch eine Freierbestrafung wäre ebenfalls kontraproduktiv, zumal die Jugendschutznorm des § 182 StGB exemplarisch dort, wo Ausbeutung Jugendlicher evident ist, bereits eine Freierbestrafung und polizeiliche Kontrollen des sog. Babystrichs ermöglichen würde, was aber nicht geschieht, so dass diese Norm in der Praxis leer läuft.

Schließlich habe ich auch Zweifel, ob man der in Polizeikreisen üblichen Einschätzung, Menschenhandel sei ein Kontrolldelikt, noch folgen kann. Alle Opferzeugen der hier besprochenen Aktenanalyse des Max-Planck-Instituts wechselten die Rolle von der Beschuldigten eines ausländerrechtlichen Straftatbestandes zur Zeugin gegen einen beschuldigten Menschenhändler. Allein dies verzerrt die Aktenrealität und lässt das proaktive Tätigwerden der Polizei in einem anderen Licht erscheinen. Es geht nur in zweiter Linie um Opferschutz, in erster Linie um sog. Banden- und Schleußerkriminalität. Mit der bereits erfolgten Öffnung der EU werden diese Anlässe seltener. Mit Wegfall des Ermittlungsparagraphen der »Förderung der Prostitution« entfallen weitere Kontrollmöglichkeiten, so dass die bisherige Strategie - auch bei leichter handhabbaren Straftatbeständen (Reform 2005) des Menschenhandels nicht weiter verfolgt werden kann. Die Lage aller Prostituierten kann nur durch eine konsequente Legalisierung verbessert werden. Strafverfolgung wäre dann auf diejenigen zu erstrecken, welche die steuerrechtlichen, gewerberechtlichen, arbeits- und mietrechtlichen Spielregeln verletzen. Die Betreiber eines Bordells und diejenige, die einen Straßenstrich organisieren, müssten sich den Regeln des sozialen Rechtsstaats unterwerfen. Nur dies könnte eine konsequente Disziplinierung kriminogener Männerbünde bewirken. Eine solche Kontrolle wäre mit Sicherheit den jetzigen Kriminalisierungsversuchen weit überlegen. Außerdem ist der Jugendschutz zu überdenken. Gerade sehr junge Prostituierte benötigen akzeptierende Sozialarbeit, gerade sie sollten nicht auf den Straßenstrich abgedrängt werden. 